\title{
SIMULATION OF THE 2DOF NONLINEAR ADAPTIVE CONTROL OF A CHEMICAL REACTOR
}

\author{
Petr Dostál, Jiří Vojtěšek, and Vladimír Bobál \\ Tomas Bata University in Zlin \\ Faculty of Applied Informatics \\ nam. T.G. Masaryka 5555, Zlín 760 01, Czech republic \\ E-mail: dostalp@fai.utb.cz
}

\section{KEYWORDS}

Chemical reactor, external linear model, nonlinear control, parameter estimation, control simulation.

\section{ABSTRACT}

The paper deals with continuous-time nonlinear adaptive control of a continuous stirred tank reactor. The control strategy is based on an application of the controller consisting of a linear and nonlinear part. The static nonlinear part is derived in the way of an inversion and exponential approximation of measured or simulated input-output data. The design of the two degrees of freedom (2DOF) dynamic linear part is based on approximation of nonlinear elements in the control loop by a continuous-time external linear model with directly estimated parameters. In the control design procedure, the polynomial approach with the pole assignment method is used. The nonlinear adaptive control is tested by simulations on the nonlinear model of the CSTR with a consecutive exothermic reaction.
\end{abstract}

\section{INTRODUCTION}

Continuous stirred tank reactors (CSTRs) are units frequently used in chemical and biochemical industry. From the system theory point of view, CSTRs belong to a class of nonlinear systems with mathematical models described by sets of nonlinear differential equations. Their models are derived and described in e.g. (Corriou 2004; Ogunnaike and Ray 1994; Schmidt 2005).

It is well known that the control of chemical reactors often represents very complex problem. The control problems are due to the process nonlinearity and high sensitivity of the state and output variables to input changes. In addition, the dynamic characteristics may exhibit a varying sign of the gain in various operating points as well as non-minimum phase behaviour. Evidently, the process with such properties is hardly controllable by conventional control methods, and, its effective control requires application some of advanced methods.

One possible method to cope with this problem exploits a linear adaptive controller with parameters computed and readjusted on the basis of recursively estimated parameters of an appropriate chosen continuous-time external linear model (CT ELM) of the process. Some results obtained by this method can be found in e.g. (Dostál et al. 2007; Dostál et al. 2009).
An effective approach to the control of CSTRs and similar processes utilizes various methods of the nonlinear control (NC). Several modifications of the NC theory are described in e.g. (Astolfi et al. 2008; Vincent and Grantham 1997; Ioannou and Fidan 2006; Zhang et al. 2000). Especially, a large class of the NC methods exploits linearization of nonlinear plants, e.g. (Huba and Ondera 2009), an application of PID controllers, e.g. (Tan et al. 2002; Bányász and Keviczky 2002) or factorization of nonlinear models of the plants on linear and nonlinear parts, e.g. (Nakamura et al. 2002; Vallery et al. 2009; Chyi-Tsong Chen1 et al. 2006; Vörös 2008; Sung and Lee 2004).

In this paper, the CSTR control strategy is based on an application of the controller consisting of a static nonlinear part (SNP) and dynamic linear part (DLP). The static nonlinear part is obtained from simulated or measured steady-state characteristic of the CSTR, its inversion, exponential approximation, and, subsequently, its differentiation. On behalf of development of the linear part, the SNP including the nonlinear model of the CSTR is approximated by a continuous-time external linear model (CT ELM). For the CT ELM parameter estimation, the direct estimation in terms of filtered variables is used, see e.g. (Rao and Unbehauen 2005; Garnier and Wang 2008). The method is based on filtration of continuous-time input and output signals where the filtered variables have in the s-domain the same properties as their nonfiltered counterparts. Then, the resulting $2 \mathrm{DOF} \mathrm{CT}$ controller is derived using the polynomial approach and pole assignment method, e.g. (Kučera 1993). The simulations are performed on a nonlinear model of the CSTR with a consecutive exothermic reaction.

\section{MODEL OF THE CSTR}

Consider a CSTR with the first order consecutive exothermic reaction according to the scheme $\mathrm{A} \stackrel{k_{1}}{\longrightarrow} \mathrm{B} \stackrel{k_{2}}{\longrightarrow} \mathrm{C}$ and with a perfectly mixed cooling jacket. Using the usual simplifications, the model of the CSTR is described by four nonlinear differential equations

$$
\frac{d c_{\mathrm{A}}}{d t}=-\left(\frac{q_{\mathrm{r}}}{V_{\mathrm{r}}}+k_{1}\right) c_{\mathrm{A}}+\frac{q_{\mathrm{r}}}{V_{\mathrm{r}}} c_{\mathrm{Af}}
$$




$$
\begin{gathered}
\frac{d c_{\mathrm{B}}}{d t}=-\left(\frac{q_{\mathrm{r}}}{V_{\mathrm{r}}}+k_{2}\right) c_{\mathrm{B}}+k_{1} c_{\mathrm{A}}+\frac{q_{\mathrm{r}}}{V_{\mathrm{r}}} c_{\mathrm{Bf}} \\
\frac{d T_{\mathrm{r}}}{d t}=\frac{h_{\mathrm{r}}}{\left(\rho c_{p}\right)_{\mathrm{r}}}+\frac{q_{\mathrm{r}}}{V_{\mathrm{r}}}\left(T_{\mathrm{rf}}-T_{\mathrm{r}}\right)+\frac{A_{\mathrm{h}} U}{V_{\mathrm{r}}\left(\rho c_{p}\right)_{\mathrm{r}}}\left(T_{\mathrm{c}}-T_{\mathrm{r}}\right) \\
\frac{d T_{\mathrm{c}}}{d t}=\frac{q_{\mathrm{c}}}{V_{\mathrm{c}}}\left(T_{c \mathrm{f}}-T_{\mathrm{c}}\right)+\frac{A_{\mathrm{h}} U}{V_{\mathrm{c}}\left(\rho c_{p}\right)_{\mathrm{c}}}\left(T_{\mathrm{r}}-T_{\mathrm{c}}\right)
\end{gathered}
$$

with initial conditions $\quad c_{\mathrm{A}}(0)=c_{\mathrm{A}}^{\mathrm{s}}, \quad c_{\mathrm{B}}(0)=c_{\mathrm{B}}^{\mathrm{s}}$, $T_{\mathrm{r}}(0)=T_{\mathrm{r}}^{\mathrm{s}}$ and $T_{\mathrm{c}}(0)=T_{\mathrm{c}}^{\mathrm{s}}$. Here, $t$ is the time, $c$ are concentrations, $T$ are temperatures, $V$ are volumes, $\rho$ are densities, $c_{p}$ are specific heat capacities, $q$ are volumetric flow rates, $A_{\mathrm{h}}$ is the heat exchange surface area and $U$ is the heat transfer coefficient. The subscripts are denoted ()$_{\mathrm{r}}$ for the reactant mixture, ()$_{\mathrm{c}}$ for the coolant, ()$_{\mathrm{f}}$ for steady-state inputs and the superscript ()$^{\mathrm{s}}$ for initial conditions. The reaction rates and the reaction heat are expressed as

$$
\begin{gathered}
k_{j}=k_{0 j} \exp \left(\frac{-E_{j}}{R T_{\mathrm{r}}}\right), j=1,2 \\
h_{\mathrm{r}}=h_{1} k_{1} c_{\mathrm{A}}+h_{2} k_{2} c_{\mathrm{B}}
\end{gathered}
$$

where $k_{0}$ are pre-exponential factors, $E$ are activation energies and $h$ are reaction entalpies. The values of all parameters, inlet values and steady-state values with used units are given in Tab. 1.

Table 1: Parameters, Steady-State Inputs and Initial Conditions.

\begin{tabular}{|ll|}
\hline$V_{\mathrm{r}}=1.2 \mathrm{~m}^{3}$ & $c_{\mathrm{pr}}=4.05 \mathrm{~kJ} \mathrm{~kg}^{-1} \mathrm{~K}^{-1}$ \\
$V_{\mathrm{c}}=0.64 \mathrm{~m}^{3}$ & $c_{\mathrm{pc}}=4.18 \mathrm{~kJ} \mathrm{~kg}^{-1} \mathrm{~K}^{-1}$ \\
$\rho_{\mathrm{r}}=985 \mathrm{~kg} \mathrm{~m}^{-3}$ & $A_{\mathrm{h}}=5.5 \mathrm{~m}^{2}$ \\
$\rho_{\mathrm{c}}=998 \mathrm{~kg} \mathrm{~m}^{-3}$ & $U=43.5 \mathrm{~kJ} \mathrm{~m}^{-2} \mathrm{~min}^{-1} \mathrm{~K}^{-1}$ \\
$k_{10}=5.616 \times 10^{16} \mathrm{~min}^{-1}$ & $E_{1} / R=13477 \mathrm{~K}$ \\
$k_{20}=1.128 \times 10^{18} \mathrm{~min}^{-1}$ & $E_{2} / R=15290 \mathrm{~K}$ \\
$h_{1}=4.8 \times 10^{4} \mathrm{~kJ} \mathrm{kmol}^{-1}$ & $h_{2}=2.2 \times 10^{4} \mathrm{~kJ} \mathrm{kmol}^{-1}$ \\
\hline$c_{\mathrm{A}}^{\mathrm{s}}=1.5796 \mathrm{kmol} \mathrm{m}^{-3}$ & $c_{\mathrm{B}}^{\mathrm{s}}=1.1975 \mathrm{kmol} \mathrm{m}^{-3}$ \\
$T_{\mathrm{r}}^{\mathrm{s}}=324.80 \mathrm{~K}$ & $T_{\mathrm{c}}^{\mathrm{s}}=306.28 \mathrm{~K}$ \\
\hline$c_{\mathrm{Af}}^{\mathrm{s}}=2.85 \mathrm{kmol} \mathrm{m}^{-3}$ & $c_{\mathrm{Bf}}^{\mathrm{s}}=0 \mathrm{kmol} \mathrm{m}^{-3}$ \\
$T_{\mathrm{rf}}^{\mathrm{s}}=323 \mathrm{~K}$ & $T_{\mathrm{cf}}^{\mathrm{s}}=293 \mathrm{~K}$ \\
$q_{\mathrm{r}}^{\mathrm{s}}=0.08 \mathrm{~m}^{3} \mathrm{~min}^{-1}$ & $q_{\mathrm{c}}^{\mathrm{s}}=0.08 \mathrm{~m}^{3} \mathrm{~min}^{-1}$ \\
\hline
\end{tabular}

In terms of the practice, only the coolant flow rate can be taken into account as the control input. As the controlled output, the reactant temperature is considered. For the control purposes, the control input $u(t)$ and the controlled output $y(t)$ are defined as deviations from steady values

$$
u(t)=q_{\mathrm{c}}(t)-q_{\mathrm{c}}^{\mathrm{s}}, \quad y(t)=T_{\mathrm{r}}(t)-T_{\mathrm{r}}^{\mathrm{s}}
$$

The dependence of the reactant temperature on the coolant flow rate in the steady-state is in Fig.1.

In subsequent control simulations, the operating interval for $q_{c}$ has been determined as

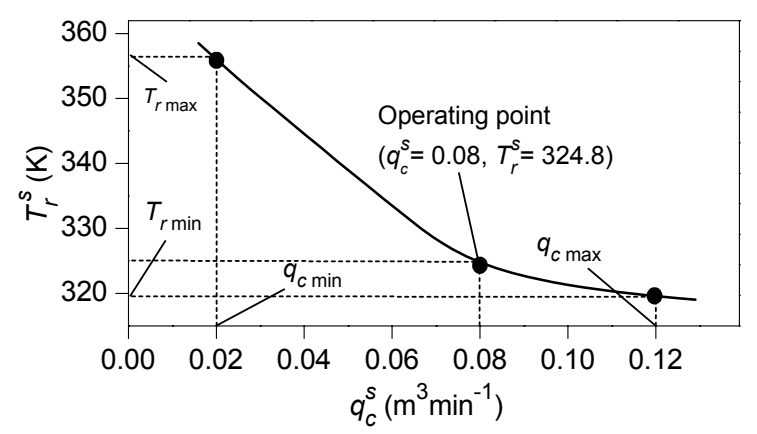

Fig. 1. Dependence of the reactant temperature on the coolant flow rate in the steady-state.

$$
q_{\mathrm{c} \text { min }} \leq q_{\mathrm{c}}(t) \leq q_{\mathrm{c} \max }
$$

With regard to the purposes of a latter steady-state characteristic approximation, the values $q_{\mathrm{cL}}$ and $q_{\mathrm{cU}}$ are established that denote the lower and upper bound of $q_{\mathrm{c}}^{\mathrm{s}}$ used for the approximation, and, $T_{\mathrm{rU}}$ and $T_{\mathrm{rL}}$ to them corresponding temperatures.

\section{CONTROLLER DESIGN}

As previously introduced, the controller consist of a static nonlinear part and a dynamic linear part as shown in Fig. 2.

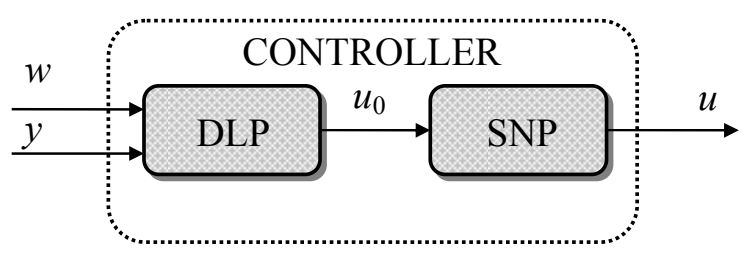

Fig. 2. The controller scheme.

The DLP creates a linear dynamic relation $u_{0}(t)=\Delta T_{\mathrm{rw}}(t)$ which represents a difference of the reactant temperature adequate to its desired value. Then, the SNP generates a static nonlinear relation betveen $u_{0}$ and a corresponding increment (decrement) of the coolant flow rate.

\section{Nonlinear Part of the Controller}

The SNP derivation appears from a simulated or measured steady-state charasteristic. The coordinates on the graph axis are defined as

$$
\gamma=\frac{q_{\mathrm{c}}^{\mathrm{s}}-q_{\mathrm{cL}}}{q_{\mathrm{cL}}}, \psi=T_{\mathrm{r}}^{\mathrm{s}}-T_{\mathrm{rL}}
$$

where $q_{\mathrm{cL}}$ is the lower bound in the interval

$$
q_{\mathrm{cL}} \leq q_{\mathrm{c}}^{\mathrm{s}} \leq q_{\mathrm{cU}}
$$

and, $T_{\mathrm{rL}}$ is the temperature corresponding to $q_{\mathrm{cU}}$.

It can be recommended to select the interval (10) slightly longer than (8). In this paper, lower and upper values in (8) and (10) were chosen $q_{\mathrm{cL}}=0.016$, 
$q_{\mathrm{c} \min }=0.02, q_{\mathrm{cmax}}=0.12$ and $q_{\mathrm{cU}}=0.13$.

In term of the practice, it can be supposed that the measured data will be affected by measurement errors. The simulated steady-state characteristic that corresponds to reality is shown in Fig. 3.

Making the replacement of coordinates, the inverse of this characteristic can be approximated by a function from the ring of polynomial, exponential, rational, eventually, by

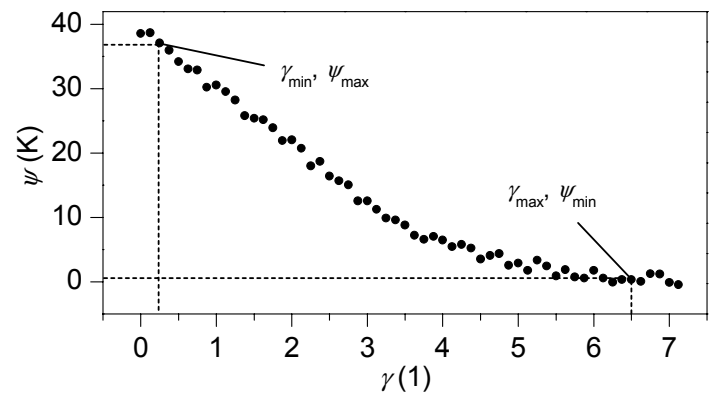

Fig. 3. Simulated characteristics $\psi=f(\gamma)$.

other type functions. Here, the second order exponential approximate function has been found in the form

$$
\begin{aligned}
\gamma= & -74071.7+2.4589 \exp \left(-\frac{\psi}{3.967}\right)+ \\
& +74076 \exp \left(-\frac{\psi}{697475}\right)
\end{aligned}
$$

The inverse characteristic together with its approximation is in Fig. 4.

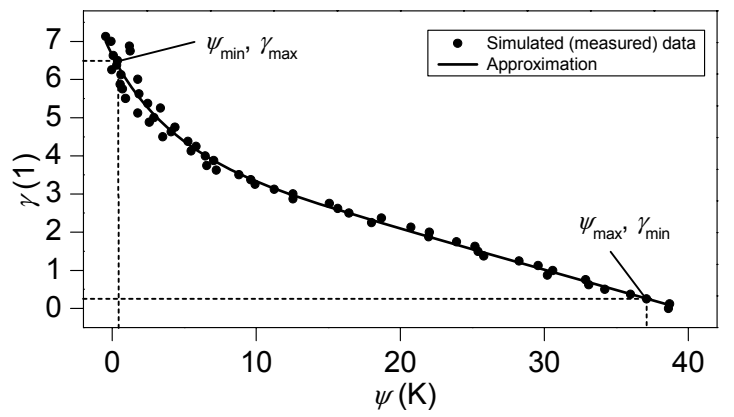

Fig. 4. Simulated and approximated inverse relation $\gamma=\varphi(\psi)$.

Now, a difference of the coolant flow rate $u(t)=\Delta q_{\mathrm{c}}(t)$ in the output of the SNP can be computed for each $T_{\mathrm{r}}$ as

$$
u(t)=\Delta q_{\mathrm{c}}(t)=q_{\mathrm{cL}}\left(\frac{d \gamma}{d \psi}\right)_{\psi\left(T_{\mathrm{r}}\right)} u_{0}(t)
$$

The derivative of the approximate function (11) takes the form

$\frac{d \gamma}{d \psi}=-0.6198 \exp \left(-\frac{\psi}{3.967}\right)-0.1062 \exp \left(-\frac{\psi}{697475}\right)$

Its plot is in Fig. 5.

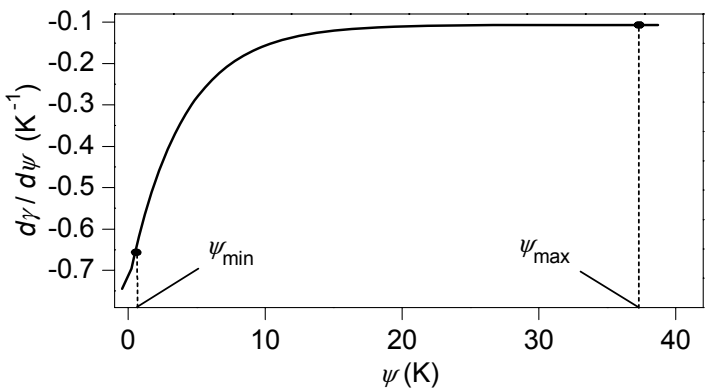

Fig. 5. Derivative of $\gamma$ with respect to $\psi$.

\section{CT External Linear Model of Nonlinear Elements}

A structure of the CT ELM of the SNP in conjuction with a nonlinear model of the CSTR was chosen on the basis of step responses simulated in a neighbourhood of the operating point $\left(q_{\mathrm{c}}^{\mathrm{s}}=0.08 \mathrm{~m}^{3} \mathrm{~min}^{-1}, T_{\mathrm{r}}^{\mathrm{s}}=324.8 \mathrm{~K}\right)$. The step responses for some step changes of $u_{0}$ are shown in Fig. 6.

For all responses, the gain of the $\mathrm{SNP}+\mathrm{CSTR}$ system has been computed as $g_{s}=\lim _{t \rightarrow \infty} \frac{y(t)}{u_{0}}$.

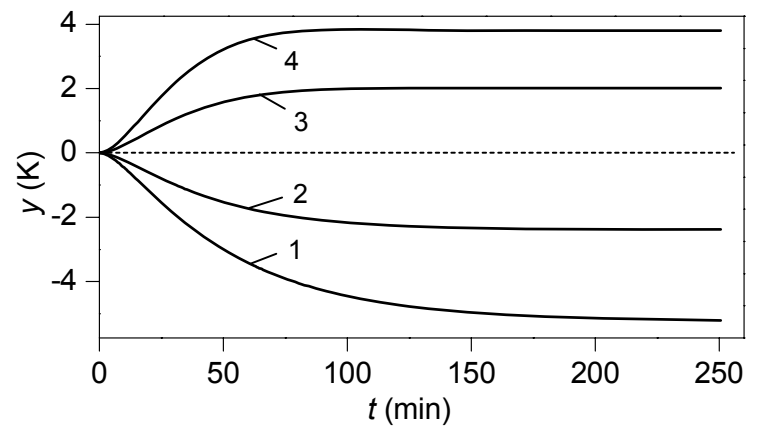

Fig. 6. Step responses of the SNP+CSTR: $1-\left(u_{0}=-4\right.$, $\left.g_{\mathrm{s}}=1.30\right), 2-\left(u_{0}=-2, \mathrm{~g}_{\mathrm{s}}=1.19\right), 3-\left(u_{0}=2\right.$, $\left.g_{\mathrm{s}}=1.01\right), 4-\left(u_{0}=4, g_{\mathrm{s}}=0.95\right)$.

Taking into account profiles of curves in Fig. 6 with zero derivatives in $t=0$, the second order CT ELM has been chosen in the form of the second order linear differential equation

$$
\ddot{y}(t)+a_{1} \dot{y}(t)+a_{0} y(t)=b_{0} u_{0}(t)
$$

or, in the transfer function representation as

$$
G(s)=\frac{Y(s)}{U_{0}(s)}=\frac{b(s)}{a(s)}=\frac{b_{0}}{s^{2}+a_{1} s+a_{0}}
$$

where $s$ is the complex variable (parameter of the Laplace transform).

\section{CT ELM Parameter Estimation}

The method of the CT ELM parameter estimation can be briefly carried out as follows.

Since the derivatives of both input and output cannot be directly measured, filtered variables $u_{0 \mathrm{f}}$ and $y_{\mathrm{f}}$ are established as the outputs of filters 


$$
\begin{gathered}
c(\sigma) u_{0 \mathrm{f}}(t)=u_{0}(t) \\
c(\sigma) y_{\mathrm{f}}(t)=y(t)
\end{gathered}
$$

where $\sigma=d / d t$ is the derivative operator, $c(\sigma)$ is a stable polynomial in $\sigma$ that fulfills the condition $\operatorname{deg} c(\sigma) \geq \operatorname{deg} a(\sigma)$.

Note that the time constants of filters must be smaller than the time constants of the process. Since the latter are unknown at the beginning of the estimation procedure, it is necessary to make the filter time constants, selected a priori, sufficiently small.

With regard to (13), the polynomial $a$ takes the concrete form $a(\sigma)=\sigma^{2}+a_{1} \sigma+a_{0}$, and, the polynomial $c$ can be chosen as $c(\sigma)=\sigma^{2}+c_{1} \sigma+c_{0}$. Subsequently, the values of the filtered variables can be computed during the control by a solution of (15) and (16) using some standard integration method.

It can be easily proved that the transfer behavior among filtered and among nonfiltered variables are equivalent. Using the $L$-transform of (15) and (16), the expressions

$$
\begin{gathered}
c(s) U_{0 \mathrm{f}}(s)=U_{0}(s)+\mu_{1}(s) \\
c(s) Y_{\mathrm{f}}(s)=Y(s)+\mu_{2}(s)
\end{gathered}
$$

can be obtained with $\mu_{1}$ and $\mu_{2}$ as polynomials of initial conditions. Substituting (17) and (18) into (14), and, after some manipulations, the relation between transforms of the filtered input and output takes the form

$$
Y_{\mathrm{f}}(s)=\frac{b(s)}{a(s)} U_{0 \mathrm{f}}(s)+M(s)=G(s) U_{0 \mathrm{f}}(s)+M(s)(
$$

where $M(s)$ is a rational function as the transform of any function $\mu(t)$ which expresses an influence of initial conditions of filtered variables.

Now, the filtered variables including their derivatives can be sampled from filters (15) and (16) in discrete time intervals $t_{k}=k T_{\mathrm{S}}, k=0,1,2, \ldots \quad$ where $T_{\mathrm{S}}$ is the sampling period. Denoting $\operatorname{deg} a=n$ and $\operatorname{deg} b=m$, the regression vector is defined as

$$
\begin{array}{r}
\boldsymbol{\Phi}\left(t_{k}\right)=\left[\begin{array}{rlll}
-y_{\mathrm{f}}\left(t_{k}\right)-y_{\mathrm{f}}^{(1)}\left(t_{k}\right) \ldots & -y_{\mathrm{f}}^{(n-1)}\left(t_{k}\right) \\
u_{0 \mathrm{f}}\left(t_{k}\right) u_{0 \mathrm{f}}^{(1)}\left(t_{k}\right) \ldots & u_{0 \mathrm{f}}^{(m)}\left(t_{k}\right) & 1
\end{array}\right]
\end{array}
$$

Now, the vector of parameters

$$
\boldsymbol{\Theta}^{T}\left(t_{k}\right)=\left[\begin{array}{llllllll}
a_{0} & a_{1} & \ldots & a_{n-1} & b_{0} & b_{1} & \ldots & b_{m}
\end{array}\right]
$$

can be estimated from the equation

$$
y_{\mathrm{f}}^{(n)}\left(t_{k}\right)=\boldsymbol{\Theta}^{T}\left(t_{k}\right) \boldsymbol{\Phi}\left(t_{k}\right)+\mu\left(t_{k}\right) .
$$

\section{Dynamic Linear Part of the Controller}

The 2DOF DLP consist of the feedback part $Q$ and the feedforward part $R$ as shown in Fig. 7 .

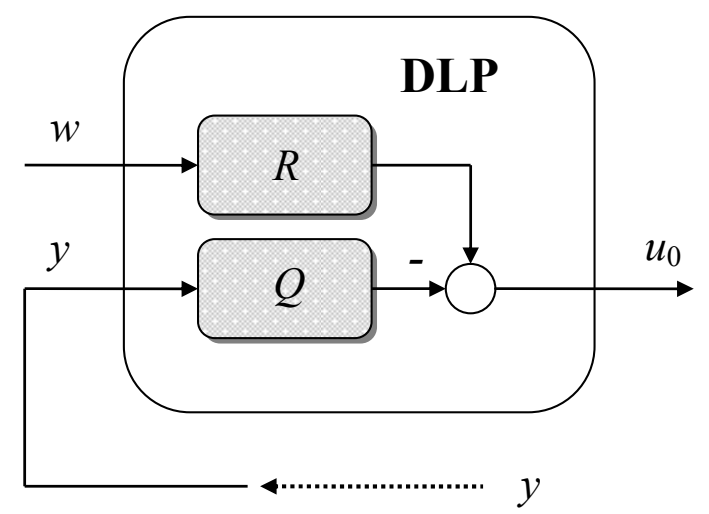

Fig. 7. Scheme of the 2DOF DLP.

In the scheme, $w$ is the reference signal, $y$ is the controlled output, and, $u_{0}$ is the input to the ELM. The reference $w$ and the disturbance $v$ that is taken into account in the next part are considered to be step functions with transforms

$$
W(s)=\frac{w_{0}}{s}, V(s)=\frac{v_{0}}{s}
$$

The transfer functions of both parts of the DLP are in forms

$$
R(s)=\frac{r(s)}{p(s)}, \quad Q(s)=\frac{q(s)}{p(s)}
$$

where $q, r$ and $p$ are coprime polynomials in $s$ fulfilling the condition of properness $\operatorname{deg} r \leq \operatorname{deg} p$ and $\operatorname{deg} q \leq \operatorname{deg} p$. For a step disturbance with the transform (23), the polynomial $p$ takes the form $p(s)=s \tilde{p}(s)$.

The controller design described in this section follows from the polynomial approach. The general conditions required to govern the control system properties are formulated as follows:

Stability of the control system, internal properness of the control system, asymptotic tracking of the reference and disturbance attenuation.

It is well known that the admissible controller results from the solution of the couple of polynomial equations

$$
\begin{gathered}
a(s) s \tilde{p}(s)+b(s) q(s)=d(s) \\
t(s) s+b(s) r(s)=d(s)
\end{gathered}
$$

with a stable polynomial $d$ on their right sides. The polynomial $t(s)$ is an auxiliary polynomial which does not enter into the controller design but it is necessary for calculation of (26).

For the transfer function (14) with $\operatorname{deg} a=2$, the degrees of controller polynomials can be derived as

$$
\operatorname{deg} q=2, \operatorname{deg} \tilde{p}=1, \operatorname{deg} r=0, \operatorname{deg} d=4
$$

and, the controller transfer functions take forms

$$
\begin{aligned}
& Q(s)=\frac{q(s)}{s \tilde{p}(s)}=\frac{q_{2} s^{2}+q_{1} s+q_{0}}{s\left(s+p_{0}\right)} \\
& R(s)=\frac{r(s)}{s \tilde{p}(s)}=\frac{r_{0}}{s\left(s+p_{0}\right)}
\end{aligned}
$$


Moreover, the equality $r_{0}=q_{0}$ can easily be obtained.

The controller parameters then follow from solutions of polynomial equations (25) and (26) and depend upon coefficients of the polynomial $d$.

In this paper, the polynomial $d$ with roots determining the closed-loop poles is chosen as

$$
d(s)=n(s)(s+\alpha)^{2}
$$

where $n$ is a stable polynomial obtained by spectral factorization

$$
a^{*}(s) a(s)=n^{*}(s) n(s)
$$

and $\alpha$ is the selectable parameter that can usually be chosen by way of simulation experiments. Note that a choice of $d$ in the form (29) provides the control of a good quality for aperiodic controlled processes. The polynomial $n$ has the form

$$
n(s)=s^{2}+n_{1} s+n_{0}
$$

with coefficients

$$
n_{0}=\sqrt{a_{0}^{2}}, \quad n_{1}=\sqrt{a_{1}^{2}+2 n_{0}-2 a_{0}} .
$$

The controller parameters can be obtained from solution of the matrix equation

$$
\left(\begin{array}{cccc}
1 & 0 & 0 & 0 \\
a_{1} & b_{0} & 0 & 0 \\
a_{0} & 0 & b_{0} & 0 \\
0 & 0 & 0 & b_{0}
\end{array}\right) \cdot\left(\begin{array}{c}
p_{0} \\
q_{2} \\
q_{1} \\
q_{0}
\end{array}\right)=\left(\begin{array}{c}
d_{3}-a_{1} \\
d_{2}-a_{0} \\
d_{1} \\
d_{0}
\end{array}\right)
$$

where

$$
\begin{aligned}
& d_{3}=n_{1}+2 \alpha, d_{2}=2 \alpha n_{1}+n_{0}+\alpha^{2} \\
& d_{1}=2 \alpha n_{0}+\alpha^{2} n_{1}, d_{0}=\alpha^{2} n_{0}
\end{aligned}
$$

Evidently, the controller parameters can be adjusted by the selectable parameter $\alpha$. The complete adaptive control system is shown in Fig. 8.

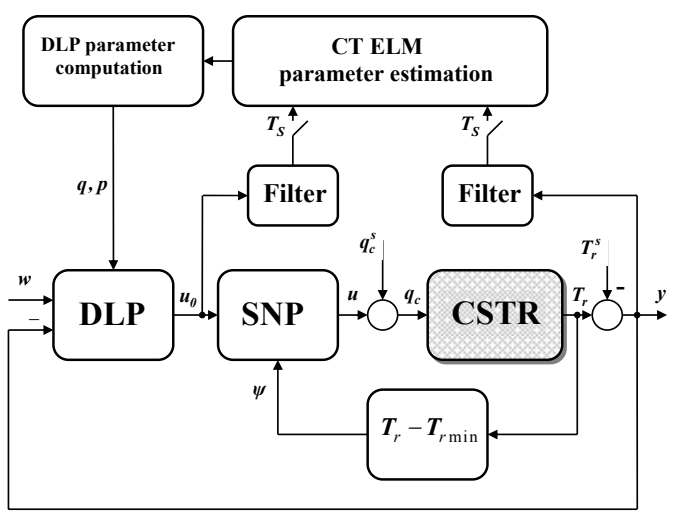

Fig. 8. Adaptive control system.

\section{CONTROL SIMULATIONS}

The control simulations were performed in a neighbourhood of the operating point

$\left(q_{\mathrm{c}}^{\mathrm{s}}=0.08 \mathrm{~m}^{3} \mathrm{~min}^{-1}, T_{\mathrm{r}}^{\mathrm{s}}=324.8 \mathrm{~K}\right)$. The filter $c(s)$ parameters were chosen as $c_{0}=0.5, c_{1}=1.5$. For the start (the adaptation phase), a P controller with a small gain was used in all simulations.

The effect of the pole $\alpha$ on the control responses is transparent from Figs. 9 and 10. Here, on the basis of precomputed simulations, three values of $\alpha$ were selected. The control results show relatively low sensitivity of the controlled output and the input signals to $\alpha$ from the selected interval. However, unsuitable selection of this parameter can lead to controlled outputs with overshoots or even to instability.

Evolution of the CT ELM parameters during control is shown in Fig. 11. In absence of disturbances, the evolution of parameters has a smooth character.

A preference of the 2DOF control system structure in comparison with the 1DOF structure with only feedback controller $Q$ can be seen in Fig. 12. There exist expressive difference between control input changes. This fact can be important in control of some reactors where expressive input changes are undesirable.

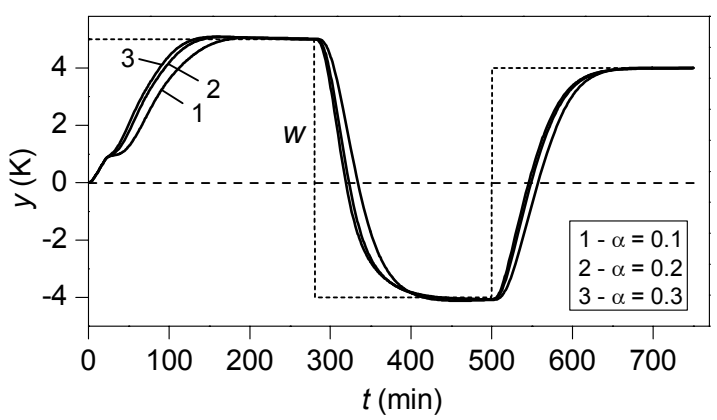

Fig. 9. Controlled output for various $\alpha$.

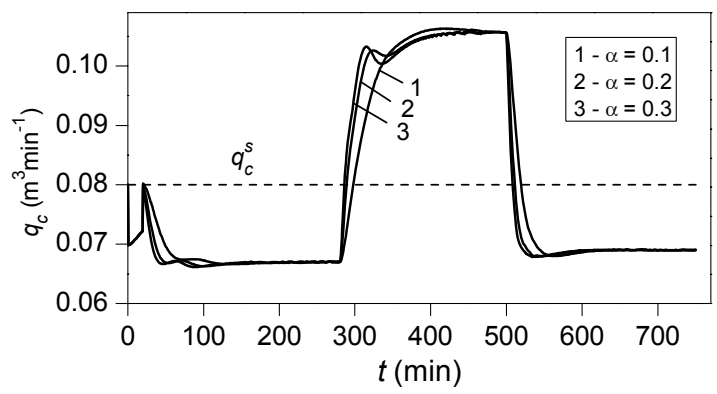

Fig. 10. Coolant flowrate for various $\alpha$.

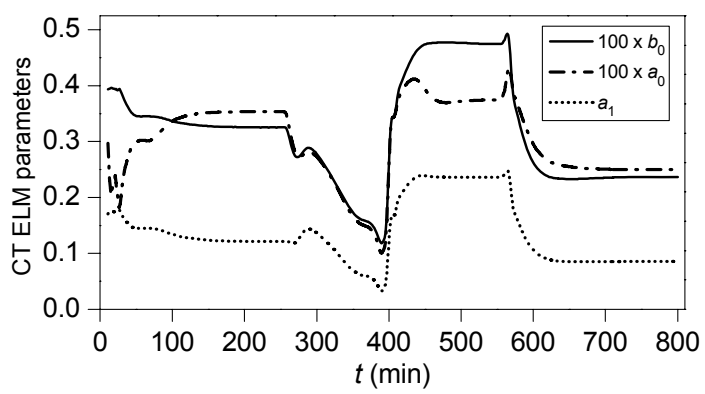

Fig. 11. Evolution of the CT ELM parameters during control. 


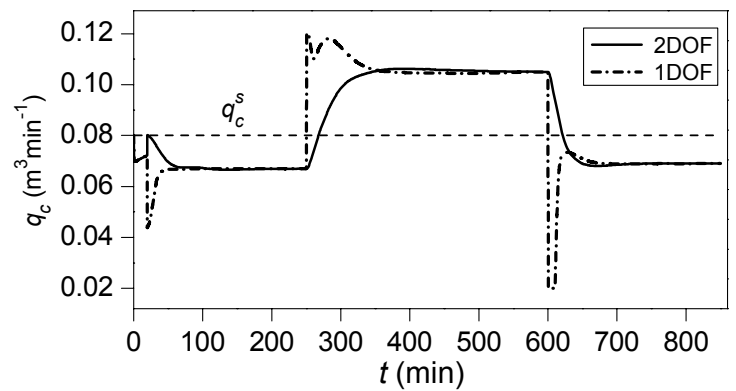

Fig. 12. Comparison of control inputs in the $1 \mathrm{DOF}$ and 2DOF structures $(\alpha=0.15)$.

A comparison of the nonlinear adaptive control with the standard adaptive control without the nonlinear part can be seen in Fig. 13. The simulations were performed for $\alpha=0.15$. The responses document priority of the nonlinear control especially for greater changes of the reference signal.

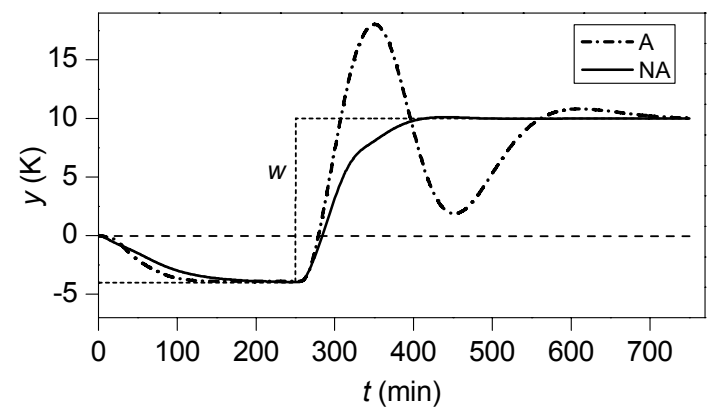

Fig. 13. Comparison of nonlinear adaptive control (NA) with standard adaptive control (A).

\section{CONCLUSIONS}

In this paper, one approach to the nonlinear continuoustime adaptive control of the reactant temperature in a continuous stirred tank reactor was proposed. The control strategy is based on a factorization of a controller into the linear and the nonlinear part. A design of the controller nonlinear part employs simulated or measured steady-state characteristics of the process and their additional modifications. Then, the system consisting of the controller nonlinear part and a nonlinear model of the CSTR is approximeted by a continuous time external linear model with parameters obtained through direct recursive parameter estimation. The resulting linear part of the continuous-time controller is considered in the 2DOF structure and derived using the polynomial approach. Tuning of its parameters is possible via closed-loop pole assignment. The presented method has been tested by computer simulation on the nonlinear model of the CSTR with a consecutive exothermic reaction. Simulation results demonstrated an applicability of the presented control strategy and its usefulness especially for greater changes of input signals in strongly nonlinear regions.

\section{ACKNOWLEDGMENT}

The authors wish to thank to the Ministry of Education of the Czech Republic (MSM7088352101) for financial support. This article was created with support of Operational Programme Research and Development for Innovations co-funded by the European Regional Development Fund (ERDF) and national budget of Czech Republic within the framework of the Centre of Polymer Systems project (reg.number: CZ.1.05/2.1.00/03.0111).

\section{REFERENCES}

Astolfi, A.; D. Karagiannis; and R. Ortega. 2008. Nonlinear and adaptive control with applications. Springer-Verlag, London.

Bányász, Cs. and L. Keviczky. 2002. "A Simple PID regulator applicable for a class of factorable nonlinear plants". In Proceedings of the American Control Conference, Anchorage, Alaska, 2354-2359.

Chyi-Tsong Chen1, Yao-Chen Chuang1 and Yao-Chen Hwang. 2006. "A simple nonlinear control strategy for chemical processes". In Proceddings of the 6th Asian Control Conference, Bali, Indonesia, 64-70.

Corriou, J.-P. 2004. Process control. Theory and applications. Springer - Verlag, London, 2004.

Dostál, P., F. Gazdoš, V. Bobál and J. Vojtěšek. 20007. "Adaptive control of a continuous stirred tank reactor by two feedback controllers." In Proceedings of the 9th IFAC Workshop Adaptation and Learning in Control and Signal Processing ALCOSP'2007, Saint Petersburg, Russia, P5-1 - P5-6.

Dostál, P., J. Vojtěšek and V. Bobál. 2009. "Simulation of adaptive control of a continuous stirred tank reactor". In Proceedings of the 23rd European Conference on Modelling and Simulation, ECMS 2009, Madrid, Spain, 625-630.

Garnier, H.; and L. Wang (eds.). 2008. Identification of continuous-time models from sampled data. SpringerVerlag, London.

Huba, M. and M. Ondera. 2009. "Simulation of nonlinear control systems represented as generalized transfer functions". In Proceedings of the European Control Conference 2009, Budapest, Hungary, 1444-1449.

Ioannou, P.; and B. Fidan. 2006. Adaptive control tutorial. SIAM, Philadelphia

Kučera, V. 1993. "Diophantine equations in control - A survey," Automatica, 29, 1361-1375.

Nakamura, M., T. Sugi and S. Goto. 2002. "Nonlinear separation model and control for a complex process realized by conventional PID controller hardware". In Proceedings of the 4th Asian Control Conference, Singapore, 274-279.

Ogunnaike, B.A.; and W.H. Ray. 1994. Process dynamics, modeling, and control. Oxford University Press, New York, 1994.

Rao, G.P. and H. Unbehauen. 2005. "Identification of continuous-time systems". IEE Proc.-Control Theory Appl., 152, 185-220.

Schmidt, L.D. 2005. The engineering of chemical reactions. Oxford University Press, New York.

Sung, S. and J. Lee. 2004. "Modeling and control of Wienertype processes". Chemical Engineering Science, 59, 15151521.

Tan, K.K., R. Ferdous and S. Huang. 2002. "Closed-loop automatic tuning of PID controller for nonlinear systems". 
In Proceedings of the 4th Asian Control Conference, Singapore, 1874-1879.

Vallery, H., M. Neumaier and M. Buss. 2009. "Anti-causal identification of Hammerstein models". In Proceedings of the European Control Conference 2009, Budapest, Hungary, 1071-1076.

Vincent,; T.L. and W.J. Grantham. 1997. Nonlinear and optimal control systems. John Wiley \& Sons, New York.

Vörös, J. 2008. "Recursive identification of Wiener systems with two segment polynomial nonlinearities". Journal of Electrical Engineering, 59(1), 40-44.

Zhang, T., S.S. Gee and C.C. Hang. 2000. "Stable adaptive control for a class of nonlinear systems using a modified Lyapunov function". IEEE Transactions on Automatic Control, 45, 129-132.

\section{AUTHOR BIOGRAPHIES}

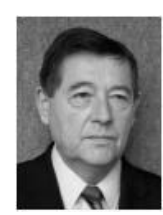

PETR DOSTÁL was born in Kněždub, Czech Republic. He studied at the Technical University of Pardubice, where he obtained his master degree in 1968 and $\mathrm{PhD}$. degree in Technical Cybernetics in 1979. In the year 2000 he became professor in Process Control. He is now head of the Department of Process Control, Faculty of Applied Informatics of the Tomas Bata University in Zlín. His research interest are modeling and simulation of continuous-time chemical processes, polynomial methods, optimal and adaptive control. You can contact him on email address dostalp@fai.utb.cz

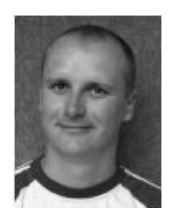

IŘí VOJTĚŠEK was born in Zlin, Czech Republic and studied at the Tomas Bata University in Zlin, where he got his master degree in chemical and process engineering in 2002 and finished his Ph.D. in Technical Cybernetics in 2007. He works as a senior lecturer in the Department of Process Control, Faculty of Applied Informatics, Tomas Bata University in Zlin. You can contact him on email address vojtesek@fai.utb.cz.

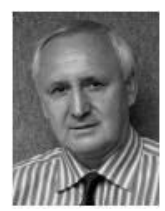

VLADIMÍR BOBÁL was born in Slavičín, Czech Republic. He graduated in 1966 from the Brno University of Technology. He received his Ph.D. degree in Technical Cybernetics at Institute of Technical Cybernetics, Slovak Academy of Sciences, Bratislava, Slovak Republic. He is now Professor in the Department of Process Control, Faculty of Applied Informatics of the Tomas Bata University in Zlín. His research interests are adaptive control systems, system identification and CAD for self-tuning controllers. You can contact him on email address bobal@fai.utb.cz 